\title{
BIOMECHANICAL CHANGES OF THE ORAL AND CRANiOfacial REgions as a Result of Altered Breathing Patterns
}

\begin{abstract}
The purpose of this report is to discuss the biomechanical changes in the oral and craniofacial regions as a result of altered breathing patterns. The increase in air pollution and allergens, resulting in allergic reactions, is often the cause of chronic nasal obstruction. The biomechancis of the oral, cranial and facial areas are intimately linked, and will be adversely affected by any change in the breathing pattern. As a result of

JORDAAN R, M PHYST (UP)'; PAPADOPOULOS $M$, MSC PHYSIO (WITS)';

nasal obstruction, the biomechanics of respiration changes to facilitate mouth breathing, and the resting position of the tongue and mandible is disrupted. The lips are parted, the freeway space and craniocervical angulation increase. The muscle activity of the posterior cervical musculature, anterior temporalis and suprahyoid muscles will be increased. The equilibrium of forces in the oral and craniofacial region is disturbed, and these abnormal forces could lead to the development of "adenoid facies", malocclusions and dysfunction over a period of time. It is important to diagnose chronic nasal obstruction and mouth breathing early and start treatment before the negative effects of the musculoskeletal system occur. A sound understanding of the biomechanical changes will enable the physiotherapist to carry out a skillful examination and plan a treatment program, based on informed decisions.
\end{abstract}

\section{KEY WORDS: BIOMECHANICS, BREATHING PATTERNS, ORO-CRANIO-FACIAL REGION, NASAL OBSTRUCTION}

\section{INTRODUCTION}

Approximately 137 years ago, a wellknown American artist George Catlin wrote about the noxious effects of mouth breathing. The title of this publication was: "The breath of life". This title was subsequently changed to "Shut your mouth and save your life". Catlin was the first to direct attention to the fact that mouth breathing can lead to facial deformity and malocclusion (abnormal alignment) of the teeth (Goldsmith and Sylvan, 1994).

The effects of oral respiration on the growth and development of craniofacial structures have been extensively studied for several decades, and have been shown to be one of the important external influences on the growth and development of

\section{CORRESPONDENCE TO:}

Mrs R Jordaan

Department of Physiotherapy

University of Pretoria

PO Box 667,

Pretoria

0001

Tel: (012) 354-2023 (W)

Tel: (012) 329-2178 (H)

Email: rjordaan@postillion.up.ac.za these structures. Knowledge regarding the neuromuscular adaptation to factors that affect function in the craniofacial region, is essential in the assessment of the long-term effects of such factors (Ono et al, 1998).

Rocabado became the first physiotherapist to analyse growth, development and soft tissue characteristics of the craniomandibular region, which he related to dentistry. From this he established a rationale for physiotherapy treatment of the maxillo-oral-facial region. He highlighted the importance of mechanical influences on the human body. Almost all tissues are sensitive to tension, compression, torsional or bending loads, and abnormal forces applied over a long period of time, may lead to skeletal deformation (Rocabado and Iglarsh, 1991).

In the orthodontic literature, many studies demonstrate the relationship between obstruction of the upper airways, malocclusions, craniofacial morphology and craniocervical posture (Hellsing et al, 1987; Özbek and Köklü, 1993; Tallgren and Solow, 1987). Wiltshire (1996), a South African orthodontist, in his study on chronic nasal obstruction and malocclusion, mentioned the increasing pre- valence of urban children in need of orthodontic treatment for malocclusions, compared to rural children. This was partly ascribed to air pollution and the possibility of increased allergen exposure in urban areas. The high degree of pollution in South Africa is a cause for increasing concern, and could have a direct effect on the incidence of malocclusions in people living in these areas. If nasal obstruction, mouth breathing and malocclusions are related to changed biomechanics of the oro-cranio-facial region, it should be a cause for increasing concern, not only for the orthodontist, but also for the physiotherapist. No mention was made in any of these studies of physiotherapy involvement in the management of these patients.

Rocabado and Iglarsh (1991) postulated that the complexity of the head, neck and maxillofacial regions requires the evaluation and treatment skills of many health professionals, including physiotherapists and dentists. The report therefore makes a valuable contribution to clinical practice, as it enables the physiotherapist to screen potential patients at an early stage in order to prevent dysfunction and symptoms from developing. This can be done by careful 
planning of suitable rehabilitation programs, based on informed decisions.

The purpose of this review article is to discuss the effects of altered breathing patterns on the biomechanics of the orocranio-facial region. Normal and abnormal breathing patterns, the causative factors of changed breathing patterns, and the biomechanical effects of changed breathing patterns will be discussed.

\section{BREATHING PATIERNS}

No two persons breathe in exactly the same manner, and no single person breathes in exactly the same way under all conditions. A variety of normal breathing patterns are used under different conditions. Before discussing altered breathing patterns, it is imperative to understand normal breathing patterns and the relevant biomechanics of the oral and craniofacial structures involved in breathing, to be able to recognize and compare changed breathing patterns and biomechanics.

\section{Normal breathing patterns}

Respiration is an important life sustaining function and jaw and tongue postures are intimately associated therewith (Wiltshire, 1996). Rocabado and Iglarsh (1991) also support this idea, but add to it, stating that the axial musculature of the spinal vertebrae is also involved in maintaining the upper airway. Furthermore, due to the fact that physiological maintenance of the airway is essential to life, it must be dynamic to accommodate the growth and development of the musculoskeletal system and its response to physical activity. It is thus clear that respiration is not an isolated function, but involves numerous other structures.

According to Ricketts (1968), normal breathing takes place through the nasal cavity, with little or no strain, while the mouth is closed. Although humans are primarily nasal breathers, everyone breathes partially through the mouth under certain physiological conditions. For the average individual, there is a transition to partial oral breathing when ventilatory exchange rates above 40 to 45 liters per minute are reached. This can happen during exercise, heavy mental concentration or even normal conversa- tion, all leading to an increase in airflow (Proffit and Fields, 1993; Ricketts, 1968).

\section{The mondibular resting position}

Respiratory needs are the primary determinant of the posture of the jaw and tongue, and of the head itself (Proffit and Fields, 1993). The natural resting position of the mandible prevents breathing through the mouth. In this position a nasal breathing pattern is present with the tongue in a resting position, the malar bone aligned with the sternum and the cranium in $15^{\circ}$ anterior rotation (Rocabado, 1998). The resting position of the mandible is the result of co-ordination between the posterior cervical muscles and the muscles that lie anterior to the cervical spine. These anterior muscles are used for inspiration, mastication, deglutition and speech. Because the mandible is contained within this group of muscles, the resting position of the mandible is dependent on the balance between these muscles (Darling et al, 1984).

In the resting position, the mandible is suspended by passive myotonic activity of the craniomandibular and the infra-mandibular musculature associated with the facial muscles. This passive activity occurs with the head held in an orthostatic position, with the malar bone of the craniofacial region aligned vertically with the sternum. In this resting position, the teeth are not in occlusion and the mandible creates an interocclusal or freeway space (space between the upper and lower teeth) (Rocabado and lglarsh, 1991).

All the stomatognatic structures are in balance in the mandibular rest position. This entails light lip contact or lips slightly apart, opposing teeth separated, jaw muscles at rest from function, mandible passively suspended against gravity, and the tongue at rest. The position of the mandible impacts directly on the position of the head on the neck, and indirectly on the entire upper body posture (Ayub et al, 1984; Rocabado and Iglarsh, 1991).

\section{The resting position of the fongue}

The resting position of the tongue against the palate occurs when the anterior part of the tongue is in contact with the rugae of the palate. The lateral margins of the tongue are contained within the lingual aspects of the maxillary bone, and the base of the tongue is in contact with the soft palate. The dorsum of the tongue is held against the hard palate by the space of negative air pressure created by the vacuum system of the tongue against the palate. This triple seal during lip closure enables the tongue to overcome the force of gravity (Ricketts, 1968; Rocabado and Iglarsh, 1991).

When the tongue assumes its normal rest position, musculature of the craniomandibular system enters a reflex relaxation stage and the mandible descends into its rest position to create the freeway space. The lips, cheeks and tongue exert a balanced internal and external force against the teeth. Therefore, normal lip seal and tongue position promote normal development of the dental alveolar region by equalizing forces applied to the teeth (Rocabado and Iglarsh, 1991).

Normal nasal breathing depends on a balanced, interactive relationship between all the head, neck and shoulder girdle components. An imbalance of one component, will have an effect on all the other components, causing an altered breathing pattern.

\section{Abnormal breathing patterns}

It is clear from the above, that the normal breathing pattern is through the nasal passages, but it is also normal to breathe through the nose and mouth under certain conditions. What is an abnormal breathing pattern?

Wenzel et al (1983), describes an increased oral involvement in respiration with obstruction of the nasal airway as an abnormal breathing pattern. According to Wiltshire (1996), when the nasal passageways prove ineffective in performing the life sustaining breathing function, the mouth exists as an emergency alternative breathing route. Rocabado and Iglarsh (1991) define mouth breathing syndrome as a habitual pattern through the mouth instead of the nose. They also mention that a combination of nasal and oral breathing is often observed which is not a normal breathing pattern. 
Respiration is most efficient with modest resistance present in the respiratory system, but increased work for nasal respiration is physiologically acceptable only to a point. With partial nose obstruction, the work associated with nasal breathing increases, and at a certain level of resistance to nasal airflow, the individual switches to partial mouth breathing. This crossover point varies among individuals. Total nasal obstruction in humans is rare, but partial nasal obstruction can occur occasionally for a short time, or in some children chronically. It is very difficult to determine what the pattern of respiration in humans really is at any given time (Proffit and Fields, 1993).

Because the respiratory need is the primary determinant of the posture of the jaw, tongue and head, it seems reasonable that an altered respiratory pattern, such as breathing through the mouth rather than the nose, can change posture of the head, jaw and tongue (Proffit and Fields, 1993).

According to Rocabado and Iglarsh (1991), a chain of body adaptations to abnormal breathing patterns leads to dysfunctional patterns. Wiltshire (1996), agrees with this statement by Rocabado and Iglarsh, saying that altered breathing patterns, such as mouth breathing, could change the equilibrial position of the tongue and jaw, which could have a significant deleterious effect on craniofacial growth and tooth positions.

These changed breathing patterns trigger the next question: What factors can contribute to change normal breathing patterns to physiologically abnormal breathing patterns?

\section{Causative factors of changed breathing patterns}

There are numerous factors that can obstruct or partially obstruct the nasal airways, either for a short time or even chronically. From the literature, it is clear that these contributing factors can be divided into three categories:

\section{Diseases of the upper respiratory tract}

Chronic respiratory obstruction can be produced by prolonged inflammation of the nasal mucosa associated with allergies, chronic respiratory infection, and asthma.
Nasal allergies and respiratory infections in early childhood are common causes of adenoidal hypertrophy, causing obstruction and restricted respiratory flow through the nasal passages. Symptoms associated with adenoidal hypertrophy and secretory otitis media, are blocked nose, mouth breathing, snoring or rhinitis (Haapaniemi, 1995; Solow et al, 1993).

\section{Developmental conditions}

The size of the posterior nasopharyngeal soft tissue, or adenoids, increase rapidly after birth. From four to six years, maximum size is attained, after which they stay the same until eight to nine years of age. Thereafter they gradually begin to involute (Haapaniemi, 1995). Any condition causing hypertrophy of the adenoids, can cause respiratory obstruction.

Rocabado and Iglarsh (1991) also mention that developmental deficiencies in the facial skeleton may predispose a patient to mouth breathing, for example a maxilla deficient in vertical height, or mandibular growth deficiencies in the mandibular head, can impair nasopharyngeal function.

\section{Physical conditions}

Extreme obesity can be a predisposing factor to a dorsal position of the tongue with a short posterior airway space between the tongue and the posterior pharyngeal wall, causing obstruction of the upper airway in adults (Solow et al, 1993). Other interesting physical conditions that can be triggering factors for changed breathing patterns, are: disturbances in the visual or proprioceptive system and cervical spine anomalies (Solow et al, 1984).

\section{The biomechanical effects of changed breathing patterns}

Changed breathing patterns will change the biomechanics of respiration. This will result in a changed mandibular posture, leading to changes in forces in the oral and craniofacial regions, and consequently this will have an effect on the head-on-neck posture, or craniocervical angulation (Miller et al, 1984; Ricketts, 1968). This chain of reactions, is due to the fact that all the craniofacial and craniocervical structures are intimately linked biomechanically.

\section{Changes in the biomechanics of respiration}

Nasal obstruction induces a change in respiratory function which involves the anterior portion of the upper respiratory tract. According to Miller et al (1984), nasal obstruction initiates a change in which the oral cavity must then serve as the major or perhaps the only pathway for periodic airflow during all respiratory demands. In adapting the oral passages for chronic respiratory work, the anterior portal is achieved by two mechanisms raising the upper lip and lowering the mandible. The posterior cavity can be widened by a protrusive action of the tongue.

In contrast to Miller's (1984) and to researchers' proposed mechanism to achieve oral breathing, Ricketts (1968) suggests that by lifting the head into extension, away from the hyo-mandibular complex, the transition from nose to mouth breathing is facilitated. According to Solow et al (1993), with obstruction of the upper airway, the craniocervical angulation increases and the possible reason could be that a changed head posture will increase the diameter of the airway, and thus reduce the airway resistance.

With mouth breathing, the accessory muscles of respiration are hyperactive. These include the sternocleidomastoid, scaleni and the pectoralis muscles. Rocabado and Iglarsh (1991) describe the typical posture associated with an increase in muscle activity of the accessory muscles of respiration, as follows: forward shoulders, depressed sternum and abnormal development of the upper thoracic region. Decreased activity of the diaphragm and hypotonicity of the abdominal musculature will perpetuate the faulty posture, characterised by protrusion of the abdominal region and an increased lumbar lordosis.

Although there are different opinions on the exact mechanisms involved in mouth breathing, it is clear that the mandible is lowered, the head is tilted into extension and the total body posture changes as a result. This will lead to changed biomechanics in the orofacial and craniofacial regions, with changed muscle activity in the associated muscles. 


\section{Chonges in the biomechonics of the oral and croniofacial regions}

Many studies agree on the adaptive changes that take place as a result of mouth breathing due to obstruction of the upper airways: the lowered position of the jaw and tongue, the parting of the lips - breaking the seal, and the extended position of the head which assists in opening the jaw by elevating the maxilla (Hellsing et al, 1987; Wenzel et al, 1985; Wiltshire, 1996).

Rocabado and Iglarsh (1991), described the sequence of oral and craniofacial changes that take place as a result of mouth breathing as follows: the resting position of the mandible is disrupted, a larger freeway space is adopted and the lips are further apart than in the nasal breather. Simultaneously, the cranium rotates posteriorly, adopting a typical forward head posture. In this head-neck position, the tongue cannot maintain a normal resting position against the palate. The tongue is pulled down onto the floor of the mouth, and slightly anterior against the posterior surface of the teeth in a thrust relation. The protrusive action of the tongue helps to open the posterior oral cavity to facilitate mouth breathing (Miller et al, 1984; Rocabado and Iglarsh, 1991).

Miller et al (1984) studied the neuromuscular changes by means of electromyography in rhesus monkeys, in which the nasal passages were obstructed with silicone plugs for two years. More than eighty percent of the monkeys demonstrated a lowered mandible, and sixty percent rhythmic tongue and upper lip movements. Certain of the craniofacial and tongue muscles were recruited and remained active throughout the two years. To change and maintain the new mandibular position requires muscle control. According to Miller et al (1984), the geniohyoid muscle is the primary suprahyoid muscle that becomes more tonically active with nasal obstruction. Other muscles recruited are the genioglossus, inferior orbicularis oris of the lower lip and the lip elevator fibers of the upper lip. It is evident that nasal obstruction causes a neuromuscular adaptation and an active change in the synaptic motor control of the craniofacial and oral muscles.
Hellsing et al (1986), demonstrated in a study on adults, that induced mouth breathing changed the electromyographic activity significantly in neck and masticatory muscles. The changed head posture associated with mouth breathing, causes the postural activity in the posterior cervical muscles to decrease. The postural activity in the anterior temporal muscle was significantly reduced to keep the mandible lowered for mouth breathing. In the suprahyoid area, increased activity was found in the anterior digastric, the geniohyoid and the genioglossus muscles. The activity in the anterior belly of the digastric muscle increased in order to depress and hold the mandible in a lowered position. The geniohyoid muscle helps to maintain the position of the hyoid bone and airway adequacy, and the genioglossus muscle helps to maintain the changed position of the tongue.

The results from the above studies demonstrate that the compensatory muscle function due to mouth breathing, causes a change in postural activity in specific neck and masticatory muscles. This stresses the fact that the mode of breathing, head posture and mandibular posture are intimately related, and that change in the one component will have an effect on the others. But what is the long term effect of the changed biomechanics of the oral and craniofacial regions?

\section{Changes in oral and craniofacial morphology}

The changed posture of the head, jaw and tongue with mouth breathing, could alter the equilibrium of pressures on the jaw and teeth, and affect jaw growth and tooth positions (Turner et al, 1997; Weinstein, 1994). Subjects who convert to mouth breathing for a short time will not have any effect on craniofacial growth. However, chronic respiratory obstruction and maintained postural changes could have a definite effect on the craniofacial growth in the growing child (Huggare, 1998). The relationship between mouth breathing, changed posture and the development of malocclusions is not clearly defined, and experimental studies have only attempted to partially clarify the enigma (Proffit and Fields, 1993; Wiltshire, 1996).
"Adenoid facies" is the popular term associated with mouth breathing, consisting of narrow facial dimensions, protruding teeth and narrowed dental arches, as well as nonfunctional lips which are separated at rest. The changed position of the tongue causes a decrease in lingual pressure on the maxillary arches, consequently losing the tongue's expansion force for normal growth and development (Rocabado and Iglarsh, 1991). With the increased pressure from the stretched cheeks due to an open mouth position, a narrowed maxillary dental arch will result (Proffit and Fields, 1993). The transverse compression of the maxillary arch causes a cross bite, and a high arched or deep palate. With cross bite, the maxillary incisors are crowded or protruded. The lips become shortened, hypertonic and nonfunctional, with less pressure on the anterior teeth which would tend to procline. A downward and backward rotation of the mandible will occur with mouth breathing, with an increased overjet and development of an anterior open bite (Proffit and Fields, 1993; Rocabado and Iglarsh, 1991; Turner et al., 1997; Wiltshire, 1996).

In the search for determinants of craniofacial development, an association between craniocervical angulation and craniofacial morphology was noticed. Among the characteristics were reduced facial prognatism, a large mandibular plane inclination and a large lower anterior facial height (Solow et al, 1994).

Solow and Kreiborg (1977) proposed a hypothesis to account for the association between airway obstruction, head posture and craniofacial morphology. They suggested that head extension causes an increase in tension, or stretching of the soft tissue layer covering the face and continuing into the investing fascia of the neck. A subsequent retrusive force on facial morphology will result.

Further consideration of the mechanism relating head posture to craniofacial morphology leads to a chain of interactions involving six factors (Figure 1). Any link in this sequence of events could be the site of primary affliction, triggering a chain of reactions (Solow and Kreiborg, 1977). 
FIGURE 1. Chain of factors relating nasal obstruction, head posture and craniofacial morphology

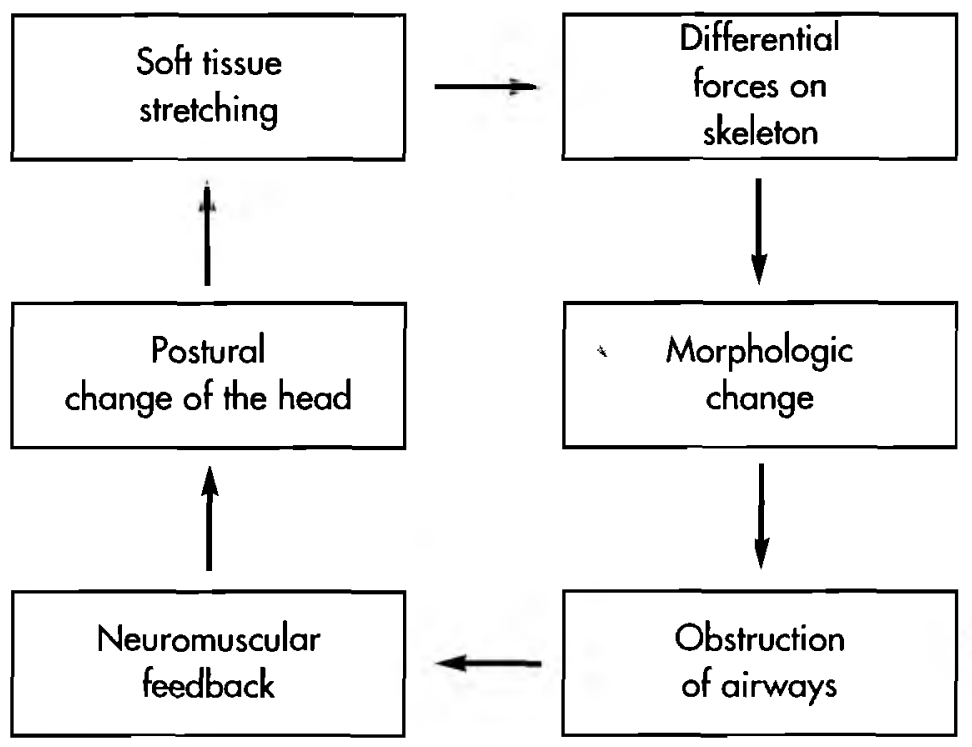

In children an example of triggering factors could be enlarged adenoid tissue, perennial allergies or asthma, causing airway obstruction, changed biomechanics of respiration, changed neuromuscular feedback and changed craniocervical posture. This will lead to stretching forces on the soft tissue. The soft tissue exerts forces on the skeletal structures as a result of the pulling effect of the stretched soft tissue on the bony elements to which they attach, leading to morphologic changes (Solow and Kreiborg, 1977; Solow et al, 1984).

Solow and Sonnesen (1998) found a clear pattern of associations between malocclusions and craniocervical posture. They described an increased craniocervical angle in children with anterior crowding of the teeth. These findings were in accordance with the soft tissue stretching hypothesis, where the increased dorsally directed soft tissue pressure causes impedement of the sagittal development of the dentalalveolar arches.

\section{Biomechanics of a changed cranioce vical angulation}

Many studies were done to determine the relationship between obstruction of the upper airways, mouth breathing and the position or posture of the head (Solow et al., 1993; Wenzel et al., 1983; Wiltshire, 1996). Solow et al (1993) examined the effect of airway obstruc- tion on craniocervical posture in a sample of adult patients with obstructive sleep apnoea. The findings did provide evidence for the hypothesis that upper airway obstruction may trigger an increase in craniocervical angulation. According to the authors, a reason for this physiological mechanism could be that such a change in posture will increase the diameter of the airway and thus reduce the airway resistance. Another reason for an increase in craniocervical angulation could be that, as observed by Ricketts (1968), an increase in the craniocervical angle will lift the head away from the hyomandibular complex, and thus facilitate the transition from nose to mouth breathing.

Studies of head posture in children with adenoidal obstruction have demonstrated an increase in craniocervical angulation or a forward head posture. After adenoidectomy, this extended head posture has been shown to change, with a decrease of up to nine degrees, of the craniocervical angle (Solow et al, 1993). Wenzel et al (1985), determined that an intranasal corticosteroid in children with asthma and perennial rhinitis, is capable of reducing nasal obstruction resistance, leading to a decrease in craniocervical angulation.

All these studies mention an increase in craniocervical angulation or head extension in relation to the cervical column. But what happens to the visual axis if the head is tilted backward into extension? The patient will be staring in an upward direction, limiting the patient's functioning.

According to Solow et al (1993), an increase in the craniocervical angulation can be mediated by extension of the head in relation to the true vertical line, or by a forward inclination of the cervical column or by a combination of both. An explanation for the different mechanisms observed, could possibly be that a need for maintaining the visual axis in its original horizontal position allows only for minor adaptations of the craniovertical angulation. A physiological requirement for a major increase in craniocervical angulation due to airway obstruction can therefore only be met by a forward inclination of the cervical column.

It is clear that the biomechanics of an increased craniocervical angulation was not studied in much detail in most of the studies.

\section{CONCLUSION}

The purpose of this report was to discuss the effects of altered breathing patterns on the biomechanics of the oro-craniofacial region. Obstruction of the upper airway, changing the breathing pattern to mouth breathing, induces a widespread chain of reactive changes. It affects the posture, biomechanics, growth and development of the orofacial, craniofacial and craniocervical regions. Not all the changes are within physiological limits and the development of stress and strain on certain neuromusculoskeletal structures, could eventually result in dysfunction.

According to Jull and Janda (1987), the importance of adequate sensory input, proprioceptive control and proper function of sensorimotor integration, has been underestimated in the pathogenes is of muscle imbalances and the pathogenesis of pain. There is a possible link between recurring headaches and craniomandibular disorders during adolescence (Capurso et al, 1997). Early diagnosis, preventative identification of the subject at risk and an interdisciplinary co-operation could prevent progression of symptoms. 
The physiotherapist must have a sound understanding of the specific reactions of tissues and growth processes to forces acting on them, as well as the principles of basic biomechanics (Leveau and Bernhardt, 1984). In planning a treatment program, it is important that the aetiological factors should always firstly be reduced, before a physiotherapeutic rehabilitative program can be introduced successfully. In the case of mouth breathing, it is important that chronic nasal obstruction be diagnosed and treated early in childhood, before negative effects on musculoskeletal growth could be induced (Wiltshire, 1996).

With good knowledge and application of these principles, clinicians can help direct the normal growth and development of the musculoskeletal system, preventing symptoms from developing in later life (Leveau and Bernhardt, 1984; Jull and Janda, 1987). This is particularly important since cranio facial growth does not cease in the late teens, but continues well into adulthood (Harris et al, 1999).

\section{REFERENCES}

Ayub E, Glasheen-Wray M B, Kraus S 1984 Head posture: a case study of the effects on the rest position of the mandible. Journal of Orthopaedic and Sports Physical Therapy 5: 179-183

Capurso U, Marini I, Vecchiet F, Bonetti G A 1997 Headache and cranio-mandibular disorders during adolescence. Journal of Clinical Pediatric Dentistry 21: 117-123

Darling D W,Kraus S, Glasheen-Wray M B 1984 Relationship of head posture and the rest position of the mandible. Journal of Prosthetic Dentistry 52: 111-115

Goldsmith J L, Sylvan E S 1994 George Catlin's concepts on mouth breathing, as presented by Dr Edward H Angle. American Journal of Orthodontics 64: 75-78

Haapaniemi J J 1995 Adenoids in schoolaged children. Journal of Laryngol Otol 109: 196-202

Harris E, Gardner R Z, Vaden J L 1999 A longitudinal cephalometric study of postorthodontic craniofacial changes. American Journal of Orthodontics and Dentofacial Orthopedics 115: $77-82$

Hellsing E, Forsberg C M, Linder-Aronson S, Sheikholeslam A 1986 Changes in postural EMG activity in the neck and masticatory muscles following obstruction of the nasal airways. European Journal of Orthodontics 8: 247-253

Hellsing E, McWilliam J, Reigo T, Spangfort E 1987 The relationship between craniofacial morphology, head posture and spinal curvature in 8,11 and 15-year old children. European Journal of Orthodontics 9: 254- 264

Huggare J 1998 Postural disorders and dentofacial morphology. ACTA Odontol Scand 56: 383-386

Jull G A, Janda V 1987 Muscles and motor control in low back pain: assessment and management. Physical Therapy of the Low Back pp253-278. Churchill Livingstone, Melbourne

Leveau B, Bernhardt D B 1984 Developmental biomechanics: effect of forces on the growth, development, and maintenance of the human body. Physical Therapy 62: 1874-1881

Miller A J, Vargervik K, Chierici G 1984 Experimentally induced neuromuscular changes during and after nasal airway obstruction. American Journal of Orthodontics 85: 385-392

Ono T, Ishiwata Y, Kuroda T 1998 Inhibition of masseteric electromyographic activity during oral respiration. American Journal of Orthodontics and Dentofacial Orthopedics 113: 518-525
Özbek M M, Köklü A 1993 Natural cervical inclination and craniofacial structure. American Journal of Orthodontics and Dentofacial Orthopedics 104: 584-591

Proffit W R, Fields H W 1993 Contemporary Orthodontics 2nd edn. ppl8 -139. Mosby Year book, Missouri

Ricketts R 1968 Respiratory obstruction syndrome. American Journal of Orthodontics 54: $459-507$

Rocabado M 1998 Temporomandibular joint and the cervical spine. Proceedings of the World Confederation of Physical Therapy Africa Congress, South Africa

Rocabado M, Iglarsh Z A 1991 Musculoskeletal approach to maxillofacial pain, pp3-137. B Lippincott Company, Philadelphia

Solow B, Kreiborg S 1977 Soft tissue stretching: a possible control factor in craniofacial morphogenesis. Scandanavian Journal of Dental Research 85: 505-507

Solow B, Ovesen J, Nielsen P W, Wildschiodtz G, Tallgren A 1993 Head posture in obstructive sleep apnoea. European Journal of Orthodontics 15: 107-114

Solow B, Siersbak-Nielsen S, Greve E 1984 Airway adequacy, head posture, and craniofacial morphology. American Journal of Orthodontics 86: 214-223

Solow B, Sonnesen L 1998 Head posture and malocclusions. European Journal of Orthodontics 20: 685-693

Tallgren A, Solow B 1987 Hyoid bone position, facial morphology and head posture in adults. European Journal of Orthodontics 9: 1-8

Turner S, Nattrass C, Sandy J R 1997 The role of soft tissues in the aetiology of malocclusion. Dental Update 24: 209-214

Weinstein S L 1994 The pediatric spine: Principles and practice, Volume 1 pp3-104. Raven Press, New York

Wenzel A, Henriksen J, Melsen B 1983 Nasal respiratory resistance: effect of intranasal corticosteroid (Budesonide) in children with asthma and perennial rhinnitis. American Journal of Orthodontics 84: 422-426

Wenzel A, Höjensgaard E, Henriksen J M 1985 Craniofacial morphology and head posture in children with asthma and perennial rhinnitis. European Journal of Orthodontics 7: 83-92

Wiltshire W A 1996 Orthodontics and chronic nasal obstruction. Current Allergy and Clinical Immunology 7: 16-18 